\title{
The pht4;1-3 mutant line contains a loss of function allele in the Fatty Acid Desaturase 7 gene caused by a remnant inactivated selection marker - A cautionary tale
}

\author{
Anders K Nilsson Corresp., 1 , Mats X Andersson ${ }^{\text {Corresp. } 1}$ \\ ${ }^{1}$ Department of Biology and Environmental Sciences, University of Gothenburg, Gothenburg, Sweden \\ Corresponding Authors: Anders K Nilsson, Mats X Andersson \\ Email address: anders.nilsson@bioenv.gu.se, mats.andersson@bioenv.gu.se
}

A striking and unexpected biochemical phenotype was found in an insertion mutant line in the model plant Arabidopsis thaliana. One of two investigated insertion mutant lines in the gene encoding the phosphate transporter PHT4;1 demonstrated a prominent loss of trienoic fatty acids, whereas the other insertion line was indistinguishable from wild type in this aspect. We demonstrate that the loss of trienoic fatty acids was due to a remnant inactive negative selection marker gene in this particular transposon tagged line, pht4;1-3. This constitutes a cautionary tale that warns of the importance to confirm the loss of this type of selection markers and the importance of verifying the relationship between a phenotype and genotype by more than one independent mutant line or alternatively genetic complementation. 
1 The pht4;1-3 mutant line contains a loss of function allele in the FATTY ACID DESATURASE 7

2 gene caused by a remnant inactivated selection marker - a cautionary tale

3

4 Anders K. Nilsson and Mats X. Andersson

5 Department of Biology and Environmental Sciences, University of Gothenburg, Box 461, SE-405 30

6 Göteborg, Sweden

7 Correspondence to: mats.andersson@bioenv.gu.se, phone: +46 317862688

9 Short title: $p h t 4 ; 1-3$ carries a FAD7 knock-out allele

11 Abstract

12 A striking and unexpected biochemical phenotype was found in an insertion mutant line in the model

13 plant Arabidopsis thaliana. One of two investigated insertion mutant lines in the gene encoding the

14 phosphate transporter PHT4;1 demonstrated a prominent loss of trienoic fatty acids, whereas the other

15 insertion line was indistinguishable from wild type in this aspect. We demonstrate that the loss of trienoic

16 fatty acids was due to a remnant inactive negative selection marker gene in this particular transposon

17 tagged line, pht4;1-3. This constitutes a cautionary tale that warns of the importance to confirm the loss of

18 this type of selection markers and the importance of verifying the relationship between a phenotype and

19 genotype by more than one independent mutant line or alternatively genetic complementation.

20 Keywords: transposon mutant, trienoic fatty acids, FAD7, PHT4;1, Arabidopsis insertion mutant

\section{Introduction}

22 Reverse genetic is a powerful tool in plant biology to establishing causal relationships between genotype and phenotype. There are numerous mutagenesis strategies, both targeted (Felippes et al. 2012; Gaj et al. 
24

2013; Yin et al. 2017) and untargeted (Alonso et al. 2003; Henikoff \& Comai 2003; McCallum et al. 2000) for generating the starting material for reverse genetics studies. Once the genetic location of a mutation has been confirmed, and the corresponding gene product is shown to be affected, the work in connecting genotype to phenotype can begin (O'Malley \& Ecker 2010). But importantly, the plant line must also be cleared of off-target mutations that may have arisen during mutagenesis. The common recommendation to circumvent this problem is to use several independent mutant lines when inferring phenotype from genotype, or alternatively, the mutant line can be functionally complemented with the wild-type copy of the gene of interest.

The PHT4;1 gene in Arabidopsis thaliana (At2g29650) encodes a phosphate transporter localized to chloroplast membranes (Guo et al. 2008; Karlsson et al. 2015; Pavon et al. 2008; Yin et al. 2015). Several loss-of-function mutant alleles for PHT4;1 have been described: pht4;1-1, pht4;1-2 and pht4;1-3 (Wang et al. 2011). The pht4;1-3 allele was isolated from a transposon insertion mutant population (Sundaresan et al. 1995; Wang et al. 2011) and has been used in at least two publications (Guo et al. 2008; Karlsson et al. 2015). The PHT4;1 transporter has not only been reported to be involved in maintaining chloroplast phosphate homeostasis (Karlsson et al. 2015) but also to play a role in plant pathogen defense by affecting salicylic acid levels (Wang et al. 2014; Wang et al. 2011). Further, plants devoid of PHT4;1 display reduced growth under standard cultivation condition, a phenotype that can be reverted by growing the pht4;1 mutants in high-phosphate conditions (Karlsson et al. 2015).

The membranes of plant chloroplast are mainly comprised of galactolipids, of which mono- and digalactosyl diacylglycerol (MGDG and DGDG, respectively) make up approximately $75 \%$ of the total chloroplastic acyl lipids (Li-Beisson et al. 2013). MGDG and DGDG are particularly enriched in trienoic fatty acids with 16 or 18 carbons in chain length (16:3 and 18:3, respectively, number of total carbon atoms:number of double bonds of the fatty acids). Arabidopsis mutants defective in the Fatty acid desaturase (FAD7) gene show reduced plastidial conversion of linoleic acid (18:2) to $\alpha$-linolenic acid (18:3), and palmitoleic acid (16:2) to hexadecatrienoic acid (16:3) (Browse et al. 1986; Iba et al. 1993). 
49 Hence, fad7 plants have a fatty acid profile skewed to contain unusually high proportion of 16:2/18:2 to $50 \quad 16: 3 / 18: 3$

51 Under phosphate limiting conditions, plants can exchange a substantial part of the plasma 52 membrane phosphoglycerolpids for galactolipids (Andersson et al. 2003). In light of these previous 53 findings, we investigated whether pht4;1 mutants are affected in leaf lipid composition. To our surprise 54 we identified a second mutation in the pht4;1-3 line located in the FAD7 locus that rendered plants 55 defective in the synthesis of trienoic fatty acids. We propose that this phenotype, not connected to 56 PHT4;1 per se, could potentially influence properties previously ascribed to absence of the phosphate 57 transporter.

Materials and methods

Plant material, growth conditions and genotyping

Arabidopsis plants were cultivated as described under short day condition (Johansson et al. 2015). The

61 pht4;1-2 and pht4;1-3 lines were a kind gift from professor Cornelia Spetea Wiklund and have been

62 previously published (Karlsson et al. 2015). The fad7-1 knock-out line (NASC ID N8042) was acquired

63 from the Nottingham Arabidopsis Stock Center (Browse et al. 1986; Scholl et al. 2000).

64 Plant DNA was extracted by heating a small leaf piece (approximately $10 \mathrm{~mm}^{2}$ ) in $750 \mu \mathrm{L}$ extraction 65 buffer (200 mM Tris- $\mathrm{HCl} \mathrm{pH} 7.5,250 \mathrm{mM} \mathrm{NaCl}, 25 \mathrm{mM}$ EDTA, $0.5 \% \mathrm{SDS})$ for $5 \mathrm{~min}$ at $95^{\circ} \mathrm{C}$. The 66 solution was left in room temp for a few hours before the DNA was precipitated through the addition of $67 \quad 750 \mu 1$ 2-propanol. The supernatant was removed after centrifugation and the pellet washed once in $70 \%$ 68 ethanol. Samples were dried in room temperature and the pellet containing DNA was reconstituted in 150 $69 \mu \mathrm{L} \mathrm{H}_{2} \mathrm{O}$.

PCRs were performed using a BioRad S1000 Thermal Cycler (Bio-Rad Laboratories, Inc., US) with

71 Titanium Taq polymerase (Clontech, Clontech Laboratories, Inc., US) or AccuPrime Pfx DNA 
72 Polymerase (Invitrogen, Life Technologies, US) according to manufacturers' instructions with primers

73 listed in table 1. PCR condition were as follows: $2 \mathrm{~min}$ initial denaturation at $95^{\circ} \mathrm{C}, 30 \mathrm{~s}$ denaturation at $7495^{\circ} \mathrm{C}, 30 \mathrm{~s}$ annealing at $58^{\circ} \mathrm{C}, 0.5-3 \mathrm{~min}$ extension at $68^{\circ} \mathrm{C}(35 \mathrm{cycles})$, and final extension for 3-5 min.

75 PCR products were analyzed by agarose gel (1-2\% agarose, Seakem LE, Lonza, Switzerland)

76 electrophoresis after staining with GelStar ${ }^{\mathrm{TM}}$ (Lonza, Switzerland).

77 The transposon in pht4;1-3 in crosses with fad7-1 or Ler were genotyped using gene specific primers pht4;1-3_FP + pht4;1-3_RP and transposon specific primers pht4;1-3_FP+CSHL_ DS5-2 (Table 1).

Extraction and quantification of lipids

The galactolipid species composition was analyzed as previously described (Nilsson et al. 2014) and total lipids fatty acid methyl esters was analyzed by GC-MS after direct transmethylation of leaf material. Leaf pieces were placed in boiling 2-propanol and supplemented with a known amount of di-nonadecanoyl phosphatidylcholine as internal standard. The samples were dried under a stream of nitrogen and transmethylated in $0.5 \mathrm{M}$ sodium methoxide in dry methanol. The fatty acid methyl esters were extracted into heptane and analyzed by GC-MS as described (Najm et al. 2017).

\section{Results and discussion}

Since PHT4;1 has been suggested to regulate phosphate transport inside the chloroplast (Karlsson et al. 2015), and since phosphate starvation is known to trigger exchange of phospholipids for galactolipids (Andersson et al. 2003), we profiled membrane lipids in the two PHT4;1 mutant alleles pht4;1-2 and pht4;1-3, and compared them to the parent wild type Ler (Figure 1). While there were no significant differences in the amount of the major membrane lipid classes, the pht4;1-3 line displayed an altered species composition of several membrane lipids. This was clear for both major thylakoid lipid classes 
95 species 34:6 and 36:6. The pht4;1-2 mutant line, on the other hand, did not differ from wild type Ler in its

96 membrane lipid composition. Analysis of fatty acid methyl esters from a total lipid extract of the pht4:1-3

97 mutant confirmed a decrease in linolenic- (18:3) and hexadecatrienoic (16:3) acids concomitant with an

98 increase in the less saturated linoleic- (18:2) and hexadecadienoic (16:2) acids (Figure 1B, Table 2). In

99 particular, 16:3 was almost completely absent from the mutant line. This phenotype is very similar to that

100 reported for mutants of the FAD7 gene (Browse et al. 1986; Iba et al. 1993), leading us to suspected that a

101 second site mutation affecting FAD7 might be present in the pht4;1-3 line.

102 To investigate this, we performed PCR on genomic DNA obtained from pht4;1-3 and wild type Ler using

103 primers spanning different regions of the FAD7 gene (Figure 2A and B). A product containing the first

104 exon and the $5^{\prime}$ UTR of FAD7 was found to be absent in reactions from the mutant line (Figure 2B).

105 Further PCR with extended elongation time reveled an approximately $2.5 \mathrm{kbp}$ insertion to be present in

106 pht4;1-3 in this region (Figure 2C). Sequencing (sequence available in Supplemental data 1) of the

107 insertion reveled it to contain an inactivated copy of the indole acetic acid hydrolase (IAAH) negative

108 selection marker used to create the transposon line (Sundaresan et al. 1995). Further, the insertion was

109 found to be located in the first exon of the FAD7 gene (Figure 2A, Supplemental data 1).

110 The $D$ s transposable element of the pht4;1-3 starter line DsG6 has previously been mapped to the FAD7

111 gene (Parinov et al. 1999). It thus appears that a part of the $D s$ element has remained in place in the

112 pht4;1-3 line due to the inability to use the negative selection based on IAAH. Rearrangements of the $D s$

113 element after transposase activation and subsequent inability to select against progeny carrying the starter

114 line cassette have previously been reported (Parinov et al. 1999).

115 To confirm that that the altered lipid profile of pht4;1-3 is indeed caused by the second insert in FAD7, a

116 genetic test was conducted. The pht4;1-3 line was crossed to fad7-1 or wild type Ler and fatty acid

117 profiles of the resulting $F_{1}$ and parental lines were analyzed (Table 2). This clearly shows that the fatty

118 acid desaturation defect in pht4;1-3 is recessive and that the $\mathrm{F}_{1}$ of pht4;1-3 and fad7-1 show an identical 
119 phenotype to their parents, supporting the inactivation of FAD7 in the pht4;1-3 line. Finally, pht4;1-3 was

120 backcrossed to Ler and the resulting $\mathrm{F}_{2}$ plants were genotyped using PCR primers for the insertion in

121 FAD7 and the previously described insertion in PHT4;1-3. Selected $\mathrm{F}_{2}$ plants homozygous for the two

122 different insertions were tested for acyl lipid fatty acid composition. This fully supported the linkage

123 between loss of trienoic fatty acids and the FAD7 insertion.

The pht4;1-3 mutant line has, as far as we could find, only been used in two publications (Karlsson et al. 2015; Wang et al. 2011). But careful scrutiny of these reports shows that in fact all but one experiments are based on the use of at least one additional allele of $p h t 4 ; 1$. The one experiment only including the pht4;1-3 allele and wild type describes the spacing of thylakoids membranes in grana stacks (Karlsson et al. 2015). Since this is a phenotype that could at least partially be attributed to the physicochemical properties of the thylakoid lipids, it seems critical that this result is confirmed with a bona fide pht4;1 mutant.

\section{Conclusions}

To conclude, this study underlines the importance that every inferred phenotype-genotype relationship is confirmed by at least two independent genetic lines of evidence, i.e. through the use of several independent knock-out or silenced mutant lines, or by genetic complementation.

\section{References}

Alonso JM, Stepanova AN, Leisse TJ, Kim CJ, Chen H, Shinn P, Stevenson DK, Zimmerman J, Barajas P, Cheuk R, Gadrinab C, Heller C, Jeske A, Koesema E, Meyers CC, Parker H, Prednis L, Ansari Y, Choy N, Deen H, Geralt M, Hazari N, Hom E, Karnes M, Mulholland C, Ndubaku R, Schmidt I, Guzman P, Aguilar-Henonin L, Schmid M, Weigel D, Carter DE, Marchand T, Risseeuw E, Brogden D, Zeko A, Crosby WL, Berry CC, and Ecker JR. 2003. Genome-wide insertional mutagenesis of Arabidopsis thaliana. Science 301:653-657. 10.1126/science.1086391 
144

145

146

147

148

149

150

151

152

153

154

155

156

157

158

159

160

161

162

163

164

165

166

167

168

169

170

171

172

173

174

175

176

177

178

179

180

181

182

183

184

185

186

187

188

189

190

191

192

193

Andersson MX, Stridh MH, Larsson KE, Liljenberg C, and Sandelius AS. 2003. Phosphate-deficient oat replaces a major portion of the plasma membrane phospholipids with the galactolipid digalactosyldiacylglycerol. FEBS Lett 537:128-132.

Browse J, McCourt P, and Somerville C. 1986. A mutant of Arabidopsis deficient in c(18:3) and c(16:3) leaf lipids. Plant Physiol 81:859-864.

Felippes FF, Wang JW, and Weigel D. 2012. MIGS: miRNA-induced gene silencing. Plant J 70:541-547. 10.1111/j.1365-313X.2011.04896.X

Gaj T, Gersbach CA, and Barbas CF, 3rd. 2013. ZFN, TALEN, and CRISPR/Cas-based methods for genome engineering. Trends in Biotechnology 31:397-405. 10.1016/j.tibtech.2013.04.004

Guo B, Jin Y, Wussler C, Blancaflor EB, Motes CM, and Versaw WK. 2008. Functional analysis of the Arabidopsis PHT4 family of intracellular phosphate transporters. New Phytol 177:889-898. 10.1111/j.1469-8137.2007.02331.x

Henikoff S, and Comai L. 2003. Single-nucleotide mutations for plant functional genomics. Annual Review of Plant Biology 54:375-401. 10.1146/annurev.arplant.54.031902.135009

Iba K, Gibson S, Nishiuchi T, Fuse T, Nishimura M, Arondel V, Hugly S, and Somerville C. 1993. A gene encoding a chloroplast omega-3 fatty acid desaturase complements alterations in fatty acid desaturation and chloroplast copy number of the fad7 mutant of Arabidopsis thaliana. J Biol Chem 268:24099-24105.

Johansson ON, Nilsson AK, Gustavsson MB, Backhaus T, Andersson MX, and Ellerstrom M. 2015. A quick and robust method for quantification of the hypersensitive response in plants. PeerJ 3:e1469. 10.7717/peerj.1469

Karlsson PM, Herdean A, Adolfsson L, Beebo A, Nziengui H, Irigoyen S, Unnep R, Zsiros O, Nagy G, Garab G, Aronsson H, Versaw WK, and Spetea C. 2015. The Arabidopsis thylakoid transporter PHT4;1 influences phosphate availability for ATP synthesis and plant growth. Plant J 84:99-110. $10.1111 /$ tpj. 12962

Li-Beisson Y, Shorrosh B, Beisson F, Andersson MX, Arondel V, Bates PD, Baud S, Bird D, Debono A, Durrett TP, Franke RB, Graham IA, Katayama K, Kelly AA, Larson T, Markham JE, Miquel M, Molina I, Nishida I, Rowland O, Samuels L, Schmid KM, Wada H, Welti R, Xu C, Zallot R, and Ohlrogge J. 2013. Acyl-lipid metabolism. Arabidopsis Book 11:e0161. 10.1199/tab.0161

McCallum CM, Comai L, Greene EA, and Henikoff S. 2000. Targeting induced local lesions IN genomes (TILLING) for plant functional genomics. Plant Physiol 123:439-442.

Najm S, Löfqvist C, Hellgren G, Engström E, Lundgren P, Hård A-L, Lapillonne A, Sävman K, Nilsson AK, Andersson MX, Smith LEH, and Hellström A. 2017. Effects of a lipid emulsion containing fish oil on polyunsaturated fatty acid profiles, growth and morbidities in extremely premature infants: A randomized controlled trial. Clinical Nutrition ESPEN 20:17-23. 10.1016/j.clnesp.2017.04.004

Nilsson AK, Johansson ON, Fahlberg P, Steinhart F, Gustavsson MB, Ellerstrom M, and Andersson MX. 2014. Formation of oxidized phosphatidylinositol and 12-oxo-phytodienoic acid containing acylated phosphatidylglycerol during the hypersensitive response in Arabidopsis. Phytochemistry 101:65-75. 10.1016/j.phytochem.2014.01.020

O'Malley RC, and Ecker JR. 2010. Linking genotype to phenotype using the Arabidopsis unimutant collection. Plant J 61:928-940. 10.1111/j.1365-313X.2010.04119.x

Parinov S, Sevugan M, Ye D, Yang WC, Kumaran M, and Sundaresan V. 1999. Analysis of flanking sequences from dissociation insertion lines: a database for reverse genetics in Arabidopsis. Plant Cell 11:2263-2270.

Pavon LR, Lundh F, Lundin B, Mishra A, Persson BL, and Spetea C. 2008. Arabidopsis ANTR1 is a thylakoid $\mathrm{Na}+$-dependent phosphate transporter: functional characterization in Escherichia coli. $J$ Biol Chem 283:13520-13527. 10.1074/jbc.M709371200

Scholl RL, May ST, and Ware DH. 2000. Seed and molecular resources for Arabidopsis. Plant Physiol 124:1477-1480. 
194

195

196

197

198

199

200

201

202

203

204

205

206

207

208

209

210

211

212

213

214

215

216

217

218

219

Sundaresan V, Springer P, Volpe T, Haward S, Jones JD, Dean C, Ma H, and Martienssen R. 1995. Patterns of gene action in plant development revealed by enhancer trap and gene trap transposable elements. Genes Dev 9:1797-1810.

Wang G, Zhang C, Battle S, and Lu H. 2014. The phosphate transporter PHT4;1 is a salicylic acid regulator likely controlled by the circadian clock protein CCA1. Front Plant Sci 5:701. $10.3389 /$ fpls.2014.00701

Wang GY, Shi JL, Ng G, Battle SL, Zhang C, and Lu H. 2011. Circadian clock-regulated phosphate transporter PHT4;1 plays an important role in Arabidopsis defense. Mol Plant 4:516-526. $10.1093 / \mathrm{mp} / \mathrm{ssr} 016$

Yin K, Gao C, and Qiu JL. 2017. Progress and prospects in plant genome editing. Nat Plants 3:17107. 10.1038/nplants.2017.107

Yin L, Vener AV, and Spetea C. 2015. The membrane proteome of stroma thylakoids from Arabidopsis thaliana studied by successive in-solution and in-gel digestion. Physiologia Plantarum 154:433446. $10.1111 / \mathrm{ppl} .12308$

\section{Figures and legends}

Figure 1. The pht4;1-3 line has a deficiency in trienoic fatty acids.

Lipids were extracted from the indicated lines and subjected to LC-MS/MS and the species distribution of MGDG and DGDG is shown (A). Mean and standard deviation of four biological replicates are shown.

Fatty acid methyl esters from wild type (Ler) (B) and pht4;1-3 (C) were analyzed by GC-MS and total ion chromatograms are shown.

Figure 2. The pht4;1-3 line contains an insertion in the FAD7 gene.

A, Gene model of FAD7 and locations of primers used for PCR. B and C, Agarose gel electrophoresis of PCR products from the indicated lines and primers. Expected fragment sizes are shown below lanes. An approximately $2.5 \mathrm{kbp}$ insertion in FAD7 in the pht4;1-3 line is seen (C). 


\section{Figure 1}

Figure 1. The pht4;1-3 line has a deficiency in trienoic fatty acids.

Lipids were extracted from the indicated lines and subjected to LC-MS/MS and the species distribution of MGDG and DGDG is shown (A). Mean and standard deviation of four biological replicates are shown. Fatty acid methyl esters from wild type (Ler) (B) and pht4;1-3 (C) were analyzed by GC-MS and total ion chromatograms are shown.

a

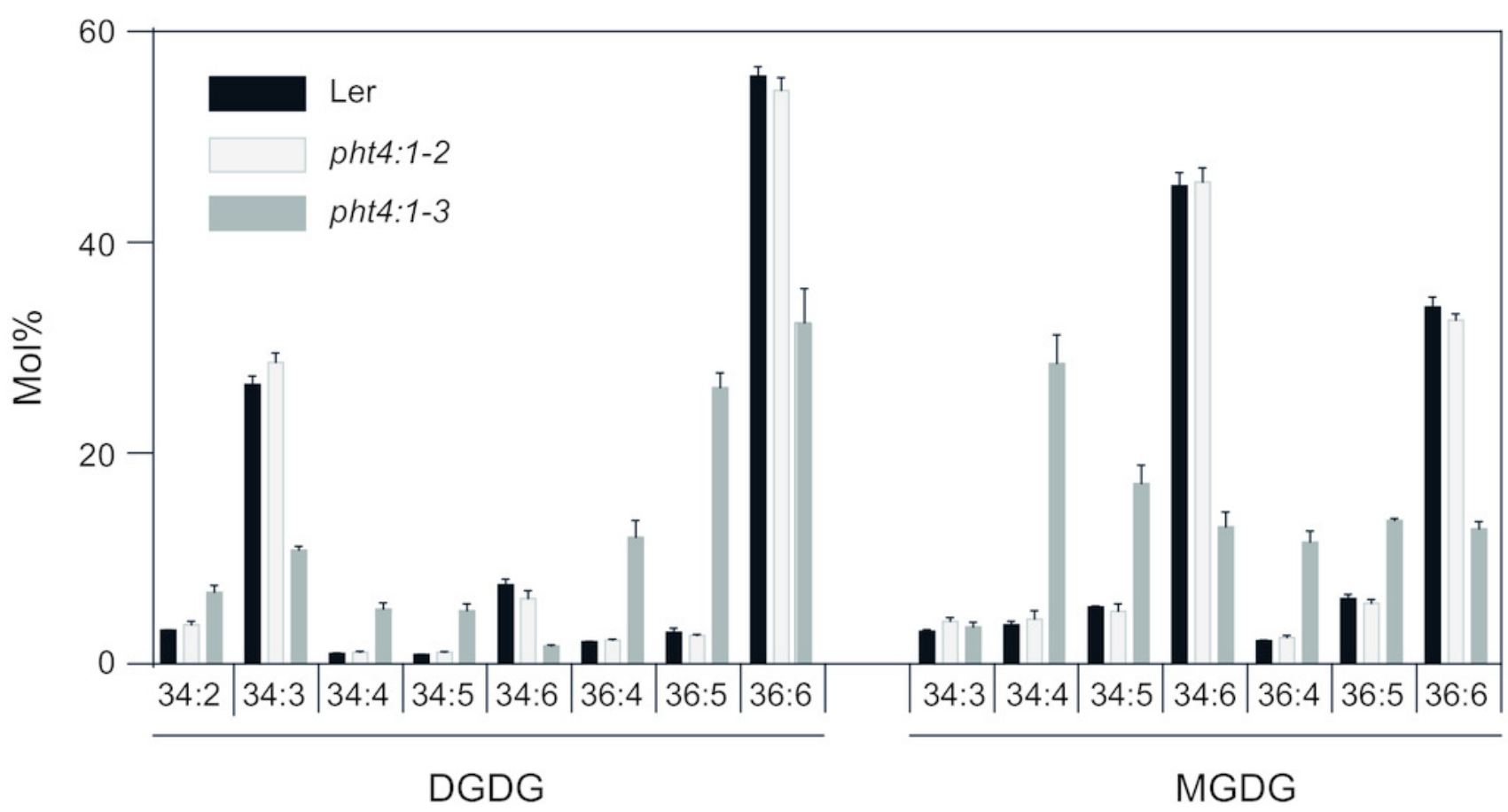

b

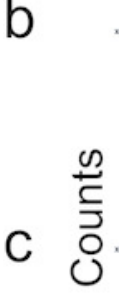

Ler

$16: 3$

$\overbrace{}^{16: 0} 16: 1$

$\overbrace{18: 0^{18: 1}}^{1}$

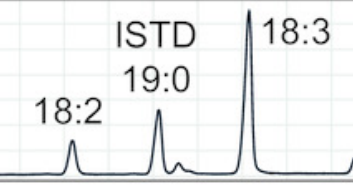

pht $4 ; 1-3$

$16: 2$

Acquisition time (min) 


\section{Figure 2}

Figure 2. The pht4;1-3 line contains an insertion in the FAD7 gene.

A, Gene model of FAD7 and locations of primers used for PCR. B and C, Agarose gel

electrophoresis of PCR products from the indicated lines and primers. Expected fragment sizes are shown below lanes. An approximately $2.5 \mathrm{kbp}$ insertion in FAD7 in the pht4;1-3 line is seen (C).

a

a Ds element

FAD7
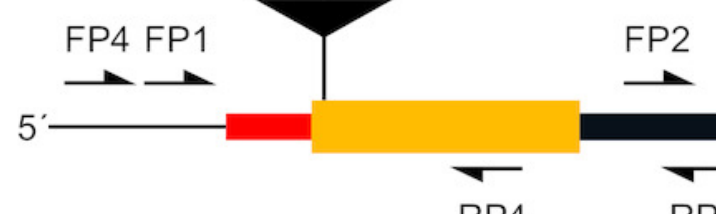

RP4

RP1

RP2

RP3

b

C
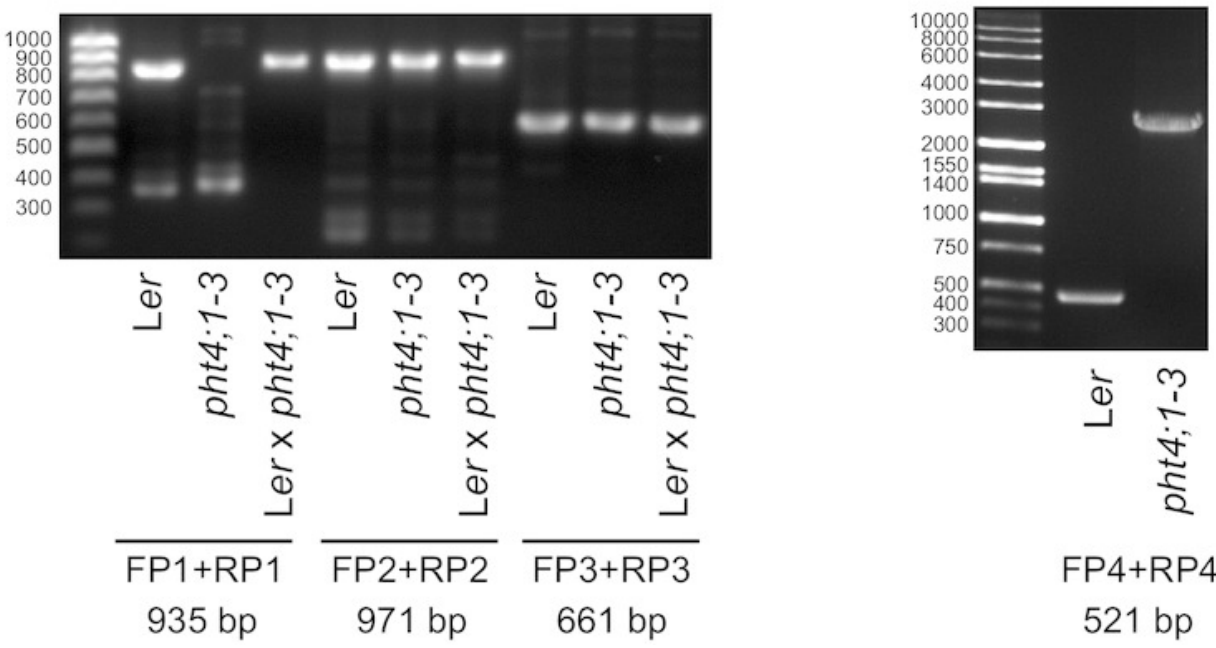

EXON

\section{UTR}

FP4+RP4

$521 \mathrm{bp}$ 
Table $\mathbf{1}$ (on next page)

Table 1. List of primer sequences used for PCR. 


\begin{tabular}{ll}
\hline Primer & Sequence \\
\hline FAD7_FP1 & AAGACATAAGCGTGCGAACC \\
FAD7_FP2 & TGTTGCTAGTAGACCAACCCA \\
FAD7_FP3 & TACCTGCATCACCATGGTCA \\
FAD7_FP4 & AATCTCACATCACACCATCACT \\
FAD7_RP1 & TCAAAGCAGATTACACAGTTGCA \\
FAD7_RP2 & TTACCTTGCCACGGTACCAA \\
FAD7_RP3 & CTAACTCTCTGGTGGGTGACA \\
FAD7_RP4 & CGCACCTGGATCGAATCTCT \\
pht4;1-3_FP & CCACCTTTGGATCCTGCCTTTAT \\
pht4;1-3_RP & ATCAACAAACCACTGATTCAACTACACTT \\
CSHL_DS5-2 & CCGTTTTGTATATCCCGTTTCCGT \\
\hline
\end{tabular}




\section{Table 2 (on next page)}

Table 2. Fatty acid profiles of pht4;1-lines.

Table 2. Fatty acid profiles of pht4;1-lines. Glycerolipid fatty acids were analyzed by GC-MS from leaf pieces obtained from the indicated lines. The following isomers are referred to for unsaturated fatty acids: 16:1, palmitoleic acid; 16:2, all-cis 9,12-hexadecadienoic acid; 16:3, all-cis 7,10,13-hexadecatrienoic acid;18:1, oleic acid; 18:2, linoleic acid; 18:3, $\alpha$-linolenic acid. Mean and standard deviation are shown based on the total number of biological replicates as indicated in the table. 


\begin{tabular}{llllllllll}
\hline Line & $\mathbf{1 6 : 0}$ & $\mathbf{1 6 : 1}$ & $\mathbf{1 6 : 2}$ & $\mathbf{1 6 : 3}$ & $\mathbf{1 8 : 0}$ & $\mathbf{1 8 : 1}$ & $\mathbf{1 8 : 2}$ & $\mathbf{1 8 : 3}$ & Replicates \\
\hline Ler & $15 \pm 0.9$ & $1.5 \pm 0.5$ & $0.5 \pm 0.1$ & $12 \pm 1.5$ & $1.2 \pm 0.4$ & $3.1 \pm 0.8$ & $14 \pm 2.0$ & $57 \pm 6.6$ & 7 \\
pht4;1-2 & $15 \pm 0.2$ & $2.0 \pm 0.1$ & $0.4 \pm 0.1$ & $14 \pm 0.2$ & $0.9 \pm 0.1$ & $2.2 \pm 0.1$ & $11 \pm 1.1$ & $55 \pm 1.2$ & 2 \\
pht4;1-3 & $14 \pm 0.9$ & $1.5 \pm 0.3$ & $9.0 \pm 1.8$ & $2.0 \pm 0.4$ & $1.1 \pm 0.3$ & $4.3 \pm 0.6$ & $30 \pm 1.6$ & $38 \pm 2.7$ & 8 \\
fad7-1 & $12 \pm 0.2$ & $1.4 \pm 0.1$ & $8.5 \pm 0.1$ & $1.7 \pm 0.1$ & $0.8 \pm 0.1$ & $4.1 \pm 0.1$ & $33 \pm 0.2$ & $39 \pm 0.1$ & 2 \\
pht4;1-3, fad7-1, $\mathrm{F}_{1}$ & $12 \pm 1.4$ & $1.2 \pm 0.1$ & $8.4 \pm 0.7$ & $1.6 \pm 0.1$ & $1.2 \pm 0.2$ & $3.8 \pm 0.3$ & $32 \pm 0.3$ & $41 \pm 2.2$ & 2 \\
pht4;1-3, Ler, $\mathrm{F}_{1}$ & $16 \pm 0.1$ & $1.4 \pm 0.1$ & $1.2 \pm 0.1$ & $9.8 \pm 0.1$ & $1.6 \pm 0.1$ & $4.6 \pm 0.4$ & $17 \pm 0.4$ & $50 \pm 0.9$ & 2 \\
pht4;1-3, Ler, $\mathrm{F}_{2}$, fad7-1 & $12 \pm 0.3$ & $1.5 \pm 0.1$ & $8.7 \pm 0.1$ & $2.0 \pm 0.1$ & $0.7 \pm 0.1$ & $5.1 \pm 0.3$ & $30 \pm 0.7$ & $39 \pm 0.8$ & 3 \\
pht4;1-3, Ler, $\mathrm{F}_{2}$, FAD7 & $13 \pm 0.2$ & $1.6 \pm 0.1$ & $1.1 \pm 0.8$ & $10 \pm 0.8$ & $0.8 \pm 0.1$ & $3.2 \pm 0.5$ & $15 \pm 2.1$ & $56 \pm 2.1$ & 6 \\
\hline
\end{tabular}

1 